\title{
Confining continuous manipulations of accelerator beam-line optics
}

\author{
Ph. Amstutz and T. Plath \\ University of Hamburg, Department of Physics, Luruper Chaussee 149, 22761 Hamburg, Germany \\ S. Ackermann, J. Bödewadt, C. Lechner, and M. Vogt \\ Deutsches Elektronen-Synchrotron DESY, Notkestraße 85, 22603 Hamburg, Germany
}

(Received 26 April 2016; published 28 April 2017)

\begin{abstract}
Altering the optics in one section of a linear accelerator beam line will in general cause an alteration of the optics in all downstream sections. In circular accelerators, changing the optical properties of any beamline element will have an impact on the optical functions throughout the whole machine. In many cases, however, it is desirable to change the optics in a certain beam-line section without disturbing any other parts of the machine. Such a local optics manipulation can be achieved by adjusting a number of additional corrector magnets that restore the initial optics after the manipulated section. In that case, the effect of the manipulation is confined in the region between the manipulated and the correcting beam-line elements. Introducing a manipulation continuously, while the machine is operating, therefore requires continuous correction functions to be applied to the correcting quadrupole magnets. In this paper, we present an approach to calculate such continuous correction functions for six quadrupole magnets by means of a homotopy method. Besides a detailed derivation of the method, we present its application to an algebraic example, as well as its demonstration at the seeding experiment sFLASH at the free-electron laser FLASH located at DESY in Hamburg.
\end{abstract}

DOI: 10.1103/PhysRevAccelBeams.20.042802

\section{INTRODUCTION}

Many applications of particle accelerators require the dynamical manipulation of the optical functions in certain regions of the beam line during operation as, for example, minimizing the $\beta$-function at the interaction point of a collider experiment, or closing a variable-gap undulator in a synchrotron light source or a free-electron laser (FEL). Changing the optical properties of a beam-line element, however, causes not only local changes in the optics but has an impact on the optical functions in all sections downstream of the adjusted element. Hence, in many cases a manipulation will result in unmatched optics in these sections and a correction is required to rematch the optics. Such manipulations are commonly conducted by interpolating between a fixed number of rematched optics in between the starting and the target optics. Following this approach, great care has to be taken to cope with the errors arising from the interpolation, see for instance Ref. [1]. In contrast to this, the method discussed in this paper allows for computing continuous correction functions, eliminating the need for interpolation.

"philipp.amstutz@desy.de

Published by the American Physical Society under the terms of the Creative Commons Attribution 3.0 License. Further distribution of this work must maintain attribution to the author $(s)$ and the published article's title, journal citation, and DOI.
Our approach is to completely confine the influence of the manipulation to a region around the adjusted elements, making the manipulation transparent for all downstream sections. This can be achieved by appropriately adjusting adjacent quadrupole magnets. Accelerator simulation codes such as ELEGANT [2] or MAD-X [3] can perform this matching and determine a suitable correction by numerically solving minimization problems. Such an approach, however, is ill-suited for calculating continuous correction functions, which are required to compensate a manipulation that is being introduced continuously, as we will discuss later. In contrast to this, we present an approach that is based on the implicit function theorem (compare for instance [4]) and allows for the determination of correction parameters as a continuous function of the introduced disturbance.

Moreover, the implementation of this correction method at the FEL user facility FLASH is presented. As depicted in Fig. 1, FLASH features two parallel undulator beam lines FLASH1 and FLASH2. Upstream of the FLASH1 main

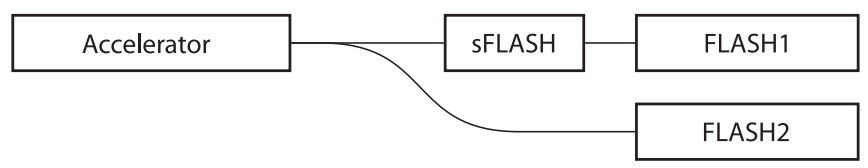

FIG. 1. Schematic layout of the FLASH facility. The superconducting linear accelerator delivers electron bunches for the FLASH1 and FLASH2 undulator beam lines and for the seeding experiment sFLASH. 
undulator the sFLASH experiment is located, which, since its installation in 2010, utilizes a variable-gap undulator system to generate seeded FEL radiation using different seeding techniques [5]. By closing this undulator in order to start seeded operation, the optics in the following FLASH1 main undulator is altered, which results in a deteriorated FEL performance. The presented method was developed to provide the means to correct the influence of the variablegap undulator and thus allow simultaneous operation of FLASH1 and sFLASH. Recently, the precise restoration of the initial optics could be verified by beam size measurements along the FLASH1 main undulator, which are presented in the final section of this paper.

\section{THE METHOD}

In linear approximation the effect of a magnetic structure on the coordinates of a particle in a $2 \mathrm{~N}$-dimensional phase space can be described by a $2 N \times 2 N$ matrix $\mathcal{M}$, called the transfer matrix [6]. It becomes apparent that any such transfer matrix is bound to be symplectic, $\mathcal{M} \in \operatorname{Sp}(2 N, \mathbb{R})$, if the energy of the particle stays constant during its passage through the structure. Furthermore it can be shown that the group of symplectic $2 N \times 2 N$ matrices is a $N(2 N+1)$ dimensional manifold [7],

$$
\operatorname{dim} \operatorname{Sp}(2 N, \mathbb{R})=N(2 N+1),
$$

with $N \in \mathbb{N}$. However, in many cases the $N$ canonical twodimensional subspaces are decoupled, so that in standard coordinates $\mathcal{M}$ takes block-diagonal form

$$
\mathcal{M}=\bigoplus_{i=1}^{N} \mathcal{A}_{i}
$$

with $\mathcal{A}_{i} \in \operatorname{Sp}(2, \mathbb{R})$. These decoupled matrices form a subgroup of $\operatorname{Sp}(2 N, \mathbb{R})$ of dimension

$$
\operatorname{dim}\left\{\bigoplus_{i=1}^{N} \mathcal{A}_{i} \mid \mathcal{A}_{i} \in \operatorname{Sp}(2, \mathbb{R})\right\}=N \operatorname{dim} \operatorname{Sp}(2, \mathbb{R})=3 N
$$

To make this distinction, we define the symbol

$$
D_{\mathcal{M}}= \begin{cases}3 N & \mathcal{M} \text { is completely decoupled } \\ N(2 N+1) & \text { otherwise }\end{cases}
$$

for a $2 N \times 2 N$ matrix $\mathcal{M}$. A transfer matrix $\mathcal{M}$ therefore has $D_{\mathcal{M}}$ degrees of freedom and hence is uniquely defined by $D_{\mathcal{M}}$ of its elements. A general six-dimensional transfer matrix, for instance, features 21 independent elements, whereas the effect of a decoupled lattice on the fourdimensional transverse phase space is given by a matrix with only six independent elements.

Every beam line can be understood as a sequence of $P$ elements, each of which is represented by a transfer matrix $\mathcal{M}_{\mathrm{n}}$. In general, the optical properties of an element can be altered during operation, e.g. by changing the current of a quadrupole magnet. Formally, we will therefore treat every transfer matrix as a function of a real experimental parameter $\rho_{n}$,

$$
\mathcal{M}_{\mathrm{n}}=\mathcal{M}_{\mathrm{n}}\left(\rho_{n}\right) .
$$

The total optical properties of a beam line are therefore given by the product of its constituent's transfer matrices

$$
\mathcal{M}: \mathbb{R}^{P} \rightarrow \mathbb{R}^{2 N \times 2 N}, \quad \mathcal{M}(\boldsymbol{\rho})=\prod_{n=0}^{P-1} \mathcal{M}_{P-n}\left(\rho_{P-n}\right) .
$$

For the further discussion, it will be helpful to divide the machine parameters $\boldsymbol{\rho}=\left(\rho_{1}, \ldots, \rho_{P}\right)$ into three pairwise disjoint subsets $\boldsymbol{\rho}=\boldsymbol{\sigma} \cup \boldsymbol{\tau} \cup \boldsymbol{v}$, the meaning of which will be illustrated in the following.

The symbol $\boldsymbol{\sigma}$ denotes those $S$ parameters that are to be varied and thereby introduce a deviation from the initial optics. Any physically realizable variation of these parameters will have to occur continuously over time and hence will be given by a continuous function,

$$
\boldsymbol{\sigma}(t)=\left(\sigma_{1}(t), \ldots, \sigma_{S}(t)\right)^{T},
$$

with the time parameter $t$ running from 0 to the point in time $t_{f}$ at which the variation is finished. In many experimental situations, the influence of this manipulation on the optics in sections subsequent to the manipulated elements is undesirable and the ability to freely change certain machine parameters without influencing the overall optical properties of a beam-line section would be of great benefit. To achieve this, the disturbance has to be corrected by another subset of $T$ machine parameters $\tau$. As we will later see, our method will be applicable to an arbitrary number $S$ of disturbance parameters but the number of correction parameters $T$ is fixed. All other parameters $\boldsymbol{v}=\boldsymbol{\rho} \backslash(\boldsymbol{\sigma} \cup \boldsymbol{\tau})$ are assumed to be constant during the compensation process. See Table I for a summary of the introduced parameters.

It will be handy to introduce a new transfer matrix function $\overline{\mathcal{M}}: \mathbb{R}^{S+T} \rightarrow \mathbb{R}^{2 N \times 2 N}$, which only depends on the dynamical parameters $\boldsymbol{\sigma}$ and $\boldsymbol{\tau}$, while the parameters $\boldsymbol{v}$ stay fixed to their initial values $\boldsymbol{v}^{0}$ :

$$
\overline{\mathcal{M}}(\boldsymbol{\sigma}, \boldsymbol{\tau})=\left.\mathcal{M}(\boldsymbol{\sigma} \cup \boldsymbol{\tau} \cup \boldsymbol{v})\right|_{\boldsymbol{v}=\boldsymbol{v}^{0}} .
$$

The purpose of the rest of this section is to develop a method to find a function $\tau(t)$ for the correction parameters

TABLE I. Symbol reference.

\begin{tabular}{ll}
\hline \hline Symbol & Description \\
\hline $\boldsymbol{\rho}$ & All machine parameters \\
$\sigma$ & Parameters to be varied \\
$\tau$ & Correction parameters \\
$\boldsymbol{v}$ & Constant parameters \\
\hline \hline
\end{tabular}


that cancels the effects caused by the manipulation of the disturbance parameters $\boldsymbol{\sigma}(t)$. This requirement on $\boldsymbol{\tau}(t)$ can be expressed by the equation

$$
\overline{\mathcal{M}}(\boldsymbol{\sigma}(t), \boldsymbol{\tau}(t))=\overline{\mathcal{M}}\left(\boldsymbol{\sigma}^{0}, \boldsymbol{\tau}^{0}\right) \quad \forall t \in\left[0, t_{f}\right],
$$

where $\boldsymbol{\sigma}^{0}=\boldsymbol{\sigma}(0)$ and $\boldsymbol{\tau}^{0}=\boldsymbol{\tau}(0)$ denote the constant initial values of the dynamical parameters. If this condition is fulfilled, the transfer matrix of the beam line is constant and hence is not affected by the changes applied to the parameters $\sigma$.

As we have seen, $\overline{\mathcal{M}}$ is a symplectic matrix and therefore has a reduced number of independent elements $D_{\overline{\mathcal{M}}}$. By defining a vector-valued function $\boldsymbol{\Phi}: \mathbb{R}^{1+T} \rightarrow \mathbb{R}^{D \overline{\mathcal{M}}}$ with the components

$$
\Phi_{i}(t, \boldsymbol{\tau})=\overline{\mathcal{M}}_{i}(\boldsymbol{\sigma}(t), \boldsymbol{\tau})-\overline{\mathcal{M}}_{i}\left(\boldsymbol{\sigma}^{0}, \boldsymbol{\tau}^{0}\right),
$$

where the $\overline{\mathcal{M}}_{i=1, \ldots, D_{\overline{\mathcal{M}}}}$ are independent elements of $\overline{\mathcal{M}}$, Eq. (4) can be written as

$$
\boldsymbol{\Phi}(t, \boldsymbol{\tau}(t))=0 \quad \forall t \in\left[0, t_{f}\right] .
$$

This is a result of the considerations in the beginning of this section: $\overline{\mathcal{M}}$ is uniquely defined by the elements $\overline{\mathcal{M}}_{i}$ and hence $\boldsymbol{\Phi}=0 \Rightarrow \overline{\mathcal{M}}=$ const.

Even for simple beam-line sections consisting only of a few elements, $\boldsymbol{\Phi}(t, \boldsymbol{\tau})$ will be a highly intricate function. In general, it is therefore not possible to solve Eq. (5) for $\boldsymbol{\tau}(t)$ by algebraic means, given an arbitrary disturbance function $\boldsymbol{\sigma}(t)$. For a given set of numerical values for the disturbance parameters it is, however, possible to find a suitable approximation for correction parameters by utilizing numerical minimization methods. This approach is readily realized with the help of existing accelerator simulation codes. To find a numerical approximation to the correction function using this method, one would have to compute the correction parameters for a number of disturbance values along $\boldsymbol{\sigma}(t)$ and interpolate. However, two successive interpolation points are not inherently correlated, as they were found by two independent numerical processes. Hence, even with a high number of interpolation points one cannot expect that this method will produce a smooth, well-behaved correction function. In contrast to this, we will derive a method that yields continuous correction functions. Loosely speaking, our approach is to track how a known root of $\boldsymbol{\Phi}$ evolves along $\boldsymbol{\sigma}(t)$, rather than to recalculate the root for each step.

The mathematical foundation of this method is the implicit function theorem. A detailed discussion of the theorem is beyond the scope of this paper but can be found in Ref. [4]. In short, the implicit function theorem states that Eq. (5) implicitly defines a unique correction function $\boldsymbol{\tau}(t)$ in a neighborhood around those machine states $(\tilde{t}, \tilde{\boldsymbol{\tau}})$, at which the Jacobian matrix is nonsingular,

$$
\operatorname{det} \frac{\partial \Phi}{\partial \boldsymbol{\tau}}(\tilde{t}, \tilde{\boldsymbol{\tau}}) \neq 0,
$$

and Eq. (5) is fulfilled

$$
\boldsymbol{\Phi}(\tilde{t}, \tilde{\boldsymbol{\tau}})=0,
$$

see Theorem 3.3.1 in Ref. [4]. For the implicit function theorem to be applicable $\boldsymbol{\Phi}$ and $\boldsymbol{\tau}$ have to be of the same dimension, $T=D_{\overline{\mathcal{M}}}$. Only then the Jacobian matrix in Eq. (6) is square and its determinant can be formed. The number of disturbance parameters $\sigma$, in contrast, is not restricted. For example, in the case of uncoupled transverse matrices, this means that a disturbance in any number of parameters may be corrected by adjusting only six correction parameters.

Let us for the moment assume that for a certain $\boldsymbol{\sigma}(t)$ the corresponding correction function $\tau(t)$ exists. By differentiating Eq. (5) with respect to $t$,

$$
\frac{\mathrm{d} \boldsymbol{\Phi}}{\mathrm{d} t}=0=\frac{\partial \boldsymbol{\Phi}}{\partial t}+\frac{\partial \boldsymbol{\Phi}}{\partial \boldsymbol{\tau}} \frac{\mathrm{d} \tau}{\mathrm{d} t}
$$

an expression for the derivative of the correction function can be obtained:

$$
\begin{aligned}
\frac{\mathrm{d} \boldsymbol{\tau}}{\mathrm{d} t} & =-\left(\frac{\partial \boldsymbol{\Phi}}{\partial \boldsymbol{\tau}}\right)^{-1} \frac{\partial \boldsymbol{\Phi}}{\partial t} \\
& =: \boldsymbol{\Psi}(t, \boldsymbol{\tau}) .
\end{aligned}
$$

This defines a system of coupled nonlinear ordinary differential equations for the components of $\boldsymbol{\tau}(t)$ that needs to be solved on the interval $\left[0, t_{f}\right]$. Assuming the lattice under consideration consists only of elements with transfer matrices that are element-wise twice continuously differentiable with respect to their experimental parameter, i.e. they are in $\mathcal{C}^{2}$, and furthermore choosing $\sigma(t) \in \mathcal{C}^{2}$, it follows from Eq. (2) that $\boldsymbol{\Phi} \in \mathcal{C}^{2}$ with respect to $t$ as well as $\tau$. Let $U \subseteq \mathbb{R}^{D \overline{\mathcal{M}}}$ be an open subset of the parameter space so that for $V=\left(-\epsilon, t_{f}+\epsilon\right) \times U$,

$$
\operatorname{det} \frac{\partial \boldsymbol{\Phi}}{\partial \boldsymbol{\tau}}(\tilde{t}, \tilde{\boldsymbol{\tau}}) \neq 0 \quad \forall(\tilde{t}, \tilde{\boldsymbol{\tau}}) \in V,
$$

with $\epsilon>0$. We see that $\boldsymbol{\Psi}(t, \boldsymbol{\tau})$ is continuously differentiable on $V$ and hence, by the Picard-Lindelöf theorem, it follows that Eq. (9) has a unique, differentiable solution, see Theorem 3.1 in Ref. [8]. We would like to note that both the implicit function theorem and the Picard-Lindelöf theorem make statements only about the local existence of the correction function in a neighborhood around $t=0$. Given the continuity of $\boldsymbol{\Psi}$, however, it can be seen by Theorem 2.1 in Ref. [8] that this local solution can be extended to a maximum forward time of existence $t_{\max }$. Here, $t$ approaching $t_{\max }$ implies $(t, \boldsymbol{\tau})$ approaching the boundary of $V$. Unfortunately it cannot be guaranteed that $t_{\max } \geq t_{f}$. 
We have therefore shifted the problem of algebraically solving the intricate system (5) for $\tau(t)$ to finding the solution of the differential equations (9). While it still will be unfeasible to find a closed form solution, this problem now lends itself readily to well-known numerical approximation methods.

However, any iterative solution method based on temporal discretization such as, for example a Runge-Kutta method, will inevitably fail at the boundaries of $U$, where the condition on the Jacobian determinant Eq. (6) fails and hence its inverse in Eq. (9) does not exist. If the correction function for a certain set of correction parameters contains such a singularity of the Jacobian, these parameters are not suitable to compensate the disturbance completely and continuously. In this case, another set of correction parameters has to be chosen.

Note that because this method preserves the transfer matrix of the manipulated section throughout the manipulation, not only the optical functions are unaffected but also the phase advance $\mu$ of the section is constant at all times,

$$
\mathcal{M}=\text { const } \Rightarrow \mu=\text { const. }
$$

This is certainly of great importance for the application to circular accelerators, as it therefore allows for constant-tune optics adjustments.

\section{AN EXAMPLE}

Symbolic transfer matrices of real-world lattices typically consist of long analytical expressions, which renders obtaining solutions to Eq. (9) very challenging. To promote better understanding of the proposed method we shall nevertheless provide the reader with an algebraic, worked out example of application. For this purpose, we will apply our method to a beam line consisting of four quadrupole magnets, each separated by a distance $l$, as shown in Fig. 2(a). To simplify the calculations in this example, we restrict the problem to preserving the optical properties in just one plane of motion and treat the quadrupole magnets as thin lenses. The symbolic $2 \times 2$ transfer matrix $\mathcal{M}$ in this plane of motion is given by

$$
\mathcal{M}=\mathcal{L}\left(f_{4}\right) \cdot \mathcal{D}(l) \cdot \mathcal{L}\left(f_{3}\right) \cdot \mathcal{D}(l) \cdot \mathcal{L}\left(f_{2}\right) \cdot \mathcal{D}(l) \cdot \mathcal{L}\left(f_{1}\right),
$$

where $\mathcal{L}(f)$ is the known transfer matrix of a thin lens with focal strength $f$, while $\mathcal{D}(l)$ represents a drift space,

$$
\mathcal{L}(f)=\left(\begin{array}{ll}
1 & 0 \\
f & 1
\end{array}\right) \quad \mathcal{D}(l)=\left(\begin{array}{ll}
1 & l \\
0 & 1
\end{array}\right) .
$$

Suppose the system is in an arbitrary initial configuration $f_{i}=f_{i}^{*}$, as for example depicted in Fig. 2(a). If now the focal strength of one lens is altered, the resulting optics will deviate from the initial case. The goal of this example is to calculate functions for the focal strengths $f_{1}, f_{3}$ and $f_{4}$ that

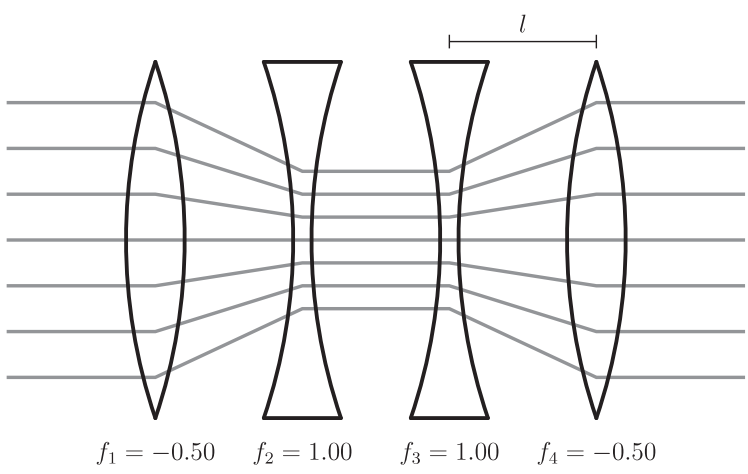

(a) Exemplary initial configuration.

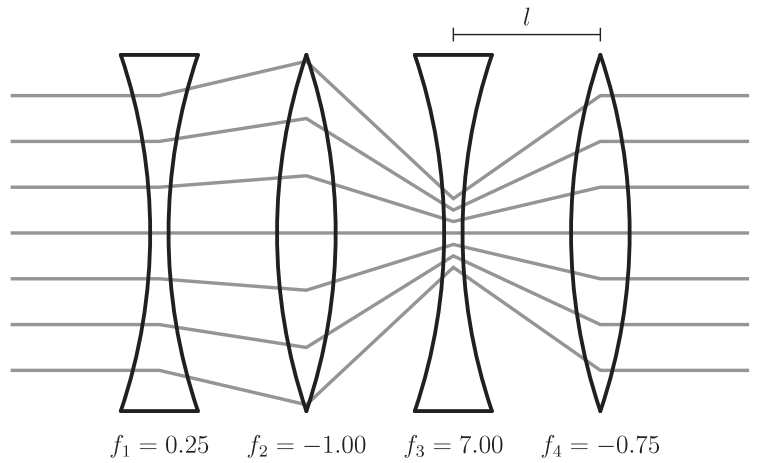

(b) Corrected state. Despite the change of sign of $f_{2}$, the overall optical properties are identical to the initial state.

FIG. 2. Schematic representation of the four-lens system in different configurations.

correct any influence variation of $f_{2}$ has on the transfer matrix of the system. Hence, using the notation introduced in Sec. II, we can identify $\boldsymbol{\sigma}=\left(f_{2}\right)$ and $\boldsymbol{\tau}=\left(f_{1}, f_{3}, f_{4}\right)$. We will investigate the case of $f_{2}$ departing linearly from its initial value,

$$
\boldsymbol{\sigma}(t)=f_{2}(t)=f_{2}^{*}+a t,
$$

at a constant rate $a \in \mathbb{R}$.

According to Eq. (1), $\mathcal{M}$ has three independent elements. Therefore, we can choose three arbitrary elements of $\mathcal{M}$ for the definition of $\boldsymbol{\Phi}(t, \boldsymbol{\tau})$. We might choose the elements in the upper triangle of $\mathcal{M}$, so that

$$
\begin{aligned}
& \boldsymbol{\Phi}(t, \boldsymbol{\tau})+\left(\mathcal{M}_{11}^{*}, \mathcal{M}_{12}^{*}, \mathcal{M}_{22}^{*}\right)^{T}=\left(\mathcal{M}_{11}, \mathcal{M}_{12}, \mathcal{M}_{22}\right)^{T} \\
& =\left(\begin{array}{c}
l\left(f_{2}\left(f_{3} l+2\right)+f_{1}\left(2 f_{3} l+f_{2} l\left(f_{3} l+2\right)+3\right)+f_{3}\right)+1 \\
l\left(2 f_{3} l+f_{2} l\left(f_{3} l+2\right)+3\right) \\
3 f_{4} l+2 f_{3} l\left(f_{4} l+1\right)+f_{2} l\left(f_{3} l+f_{4} l\left(f_{3} l+2\right)+1\right)+1
\end{array}\right),
\end{aligned}
$$

where $\mathcal{M}^{*}$ denotes the transfer matrix evaluated at the initial parameters $f_{i}=f_{i}^{*}$, and the only explicit time dependence is in $f_{2}=f_{2}(t)$.

In this case, Eq. (9) yields the following differential equations: 


$$
\frac{\mathrm{d} \boldsymbol{\tau}}{\mathrm{d} t}=\left(\frac{\mathrm{d} f_{1}}{\mathrm{~d} t}, \frac{\mathrm{d} f_{3}}{\mathrm{~d} t}, \frac{\mathrm{d} f_{4}}{\mathrm{~d} t}\right)^{T}=-\left(\frac{\partial \boldsymbol{\Phi}}{\partial \boldsymbol{\tau}}\right)^{-1} \frac{\partial \boldsymbol{\Phi}}{\partial t}=\left(\begin{array}{c}
-\frac{a\left(f_{3} l+2\right)}{\left(f_{2} l+2\right)\left(2 f_{3} l+f_{2} l\left(f_{3} l+2\right)+3\right)} \\
-\frac{a\left(f_{3} l+2\right)}{f_{2} l+2} \\
\frac{a}{2 f_{3} l+f_{2} l\left(f_{3} l+2\right)+3}
\end{array}\right) .
$$

The solutions to these equations for the initial conditions $f_{i}(0)=f_{i}^{*}$ are

$$
\begin{aligned}
& f_{1}(t)=\frac{f_{1}^{*}\left(2 f_{3}^{*} l+f_{2}^{*} l\left(f_{3}^{*} l+2\right)+3\right)\left(a l t+f_{2}^{*} l+2\right)-a t\left(f_{3}^{*} l+2\right)}{\left(2 f_{3}^{*} l+f_{2}^{*} l\left(f_{3}^{*} l+2\right)+3\right)\left(a l t+f_{2}^{*} l+2\right)} \\
& f_{3}(t)=\frac{f_{3}^{*}\left(f_{2}^{*} l+2\right)-2 a t}{a l t+f_{2}^{*} l+2} \\
& f_{4}(t)=\frac{a t}{2 f_{3}^{*} l+f_{2}^{*} l\left(f_{3}^{*} l+2\right)+3}+f_{4}^{*} .
\end{aligned}
$$

For a given point in time $t$ these functions yield values for the remaining focal strengths, so that the optical properties of the system are equivalent to those of the initial setting $f_{i}^{*}$. In Fig. 3 these functions are plotted for the initial configuration shown in Fig. 2(a), with $a=-2$. The resulting state of the system at $t=1$ is illustrated in Fig. 2(b).

It can be seen that the first two functions have a pole at $t=-\left(f_{2}^{*} l+2\right) /(a l)$, independent on the initial condition. At this point the Jacobian determinant in Eq. (6) vanishes and no continuous correction beyond this point is possible.

\section{IMPLEMENTATION AT SFLASH}

The superconducting linear accelerator FLASH at DESY in Hamburg delivers trains of electron bunches into two

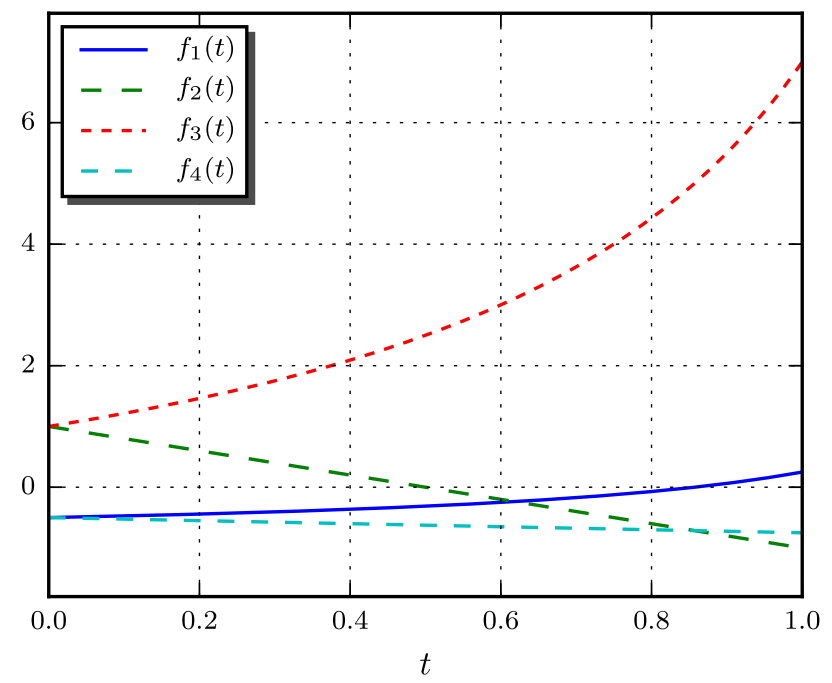

FIG. 3. Plots of the correction functions for the initial configuration shown in Fig. 2(a) and $a=-2$. parallel undulator beam lines, FLASH1 and FLASH2 [9]. Upstream of the FLASH1 main undulator an experimental setup for FEL seeding (sFLASH) is located, see Fig. 1. It features four variable-gap undulator modules and thus is the ideal test bench for the study of several different seeding schemes [5].

Closing the seeding undulator intensifies its natural focusing effect and will therefore impact the beam optics further downstream of this undulator and in particular at the entrance of the main undulator. At lower beam energies the change in the $\beta$-function can easily be in the order of several tens of percent so that the matching into the FODO structure of the main undulator is corrupted. The result is a significantly deteriorated FEL performance. Therefore, the disturbance introduced by the seeding undulator has to be compensated by adjusting the field strength of appropriate quadrupole magnets.

Initially, the presented method was developed to correct for the influence of the variable-gap undulator used by the seeding experiment at FLASH. Due to their magnetic structure, planar undulators exhibit a systematic focusing effect in the vertical plane (here, denoted by $y$ ), perpendicular to their pole faces. For our purpose, the additional weak defocusing in the horizontal direction $(x)$, stemming from the finite width of real-world undulators, can be neglected. Employing the matrix formalism introduced above, the effect of an undulator of length $l$ and undulator period $\lambda_{\mathrm{u}}$ on a particle's phase space trajectory $u=\left(x, x^{\prime}, y, y^{\prime}\right)^{T}$ is represented by the transfer matrix [10],

$$
\mathcal{M}_{\text {und }}=\left(\begin{array}{cccc}
1 & l & 0 & 0 \\
0 & 1 & 0 & 0 \\
0 & 0 & \cos \sqrt{\kappa} l & \frac{\sin \sqrt{\kappa} l}{\sqrt{\kappa}} \\
0 & 0 & -\sqrt{\kappa} \sin \sqrt{\kappa} l & \cos \sqrt{\kappa} l
\end{array}\right) \text {, }
$$




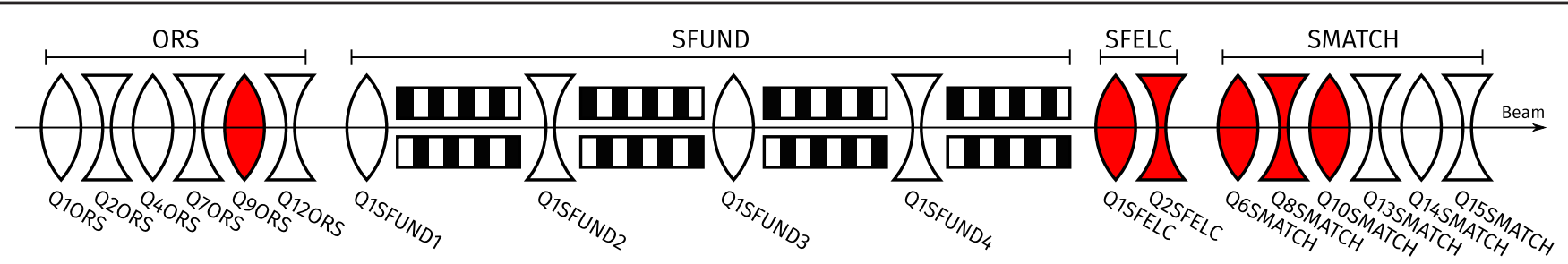

FIG. 4. Positions of the quadrupole magnets close to the sFLASH undulator. Note that the magnets in the SFUND section cannot be used as correctors. Magnets used in the example shown in Fig. 5 are highlighted in red. The drawing is not to scale.

where $\kappa$ is the focusing strength

$$
\kappa \approx 2\left(\frac{K \pi}{\gamma \lambda_{\mathrm{u}}}\right)^{2}
$$

defined by the undulator parameter $K$ and $\gamma$, the particle's Lorentz factor. By varying the value of the gap, the $K$ parameter of the undulator system used by the sFLASH experiment can be set to a value between 0 and 2.72 [11], introducing the undesirable focusing effect. We therefore identify $K$ as the disturbance parameter, $\boldsymbol{\sigma}=(K)$.

Our implementation utilizes the computer algebra system MATHEMATiCA [12], which allows for calculating the required symbolic transfer matrix according to Eq. (2), as well as setting up the differential equations (9), the numerical solution of which is then found by means of a basic Runge-Kutta algorithm. There are 14 quadrupole magnets in the vicinity of the sFLASH undulator (see Fig. 4) that are included in the MATHEMATICA model and are available as correctors. FLASH features a transversely decoupled lattice, so that exactly six of these magnets need to be chosen as the correction parameters $\tau$. Hence, there are $\left(\begin{array}{c}14 \\ 6\end{array}\right)=3003$ combinations of corrector magnets to choose from.

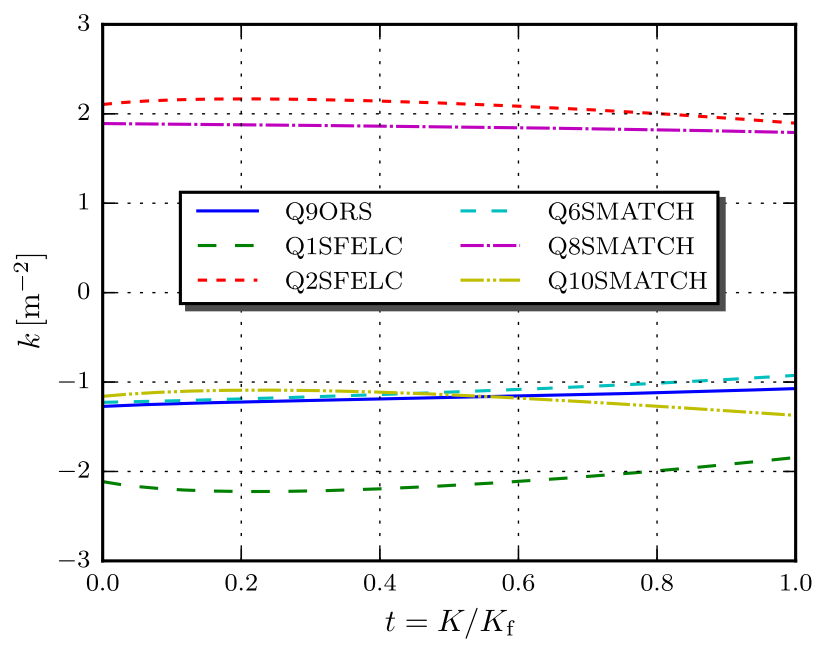

FIG. 5. Example of a correction function calculated using the presented method. Here, the strengths $k$ of the six selected quadrupole magnets (see legend) are used as correction parameters to compensate for the optics disturbance caused by the sFLASH undulator being closed from $K=0$ to a final value of $K=K_{\mathrm{f}}=2.63$ at a beam energy of $693 \mathrm{MeV}$.
Investigation shows that a lot of these combinations are prone to produce solutions featuring impracticably high changes in the quadrupole strengths or solutions containing a singularity, as mentioned above. However, we were able to identify a set of known good corrector combinations for optics similar to the standard optics used in nominal SASE operation. An example of the correction functions that are obtained by this method is shown in Fig. 5 .

Surely, for any given manipulation, correction functions exist which involve more than six corrector magnets. In that case, the solution of Eq. (5) is no longer unique because, as we have seen, solutions can be constructed using any combination of six of the magnets and leave the others constant, which results in our method being not directly applicable. However, any number of additional constraints, other than those given by the constancy of the transfer matrix, can easily be incorporated into our method by appending them to $\boldsymbol{\Phi}$ in Eq. (5) and selecting the same number of additional correction parameters.
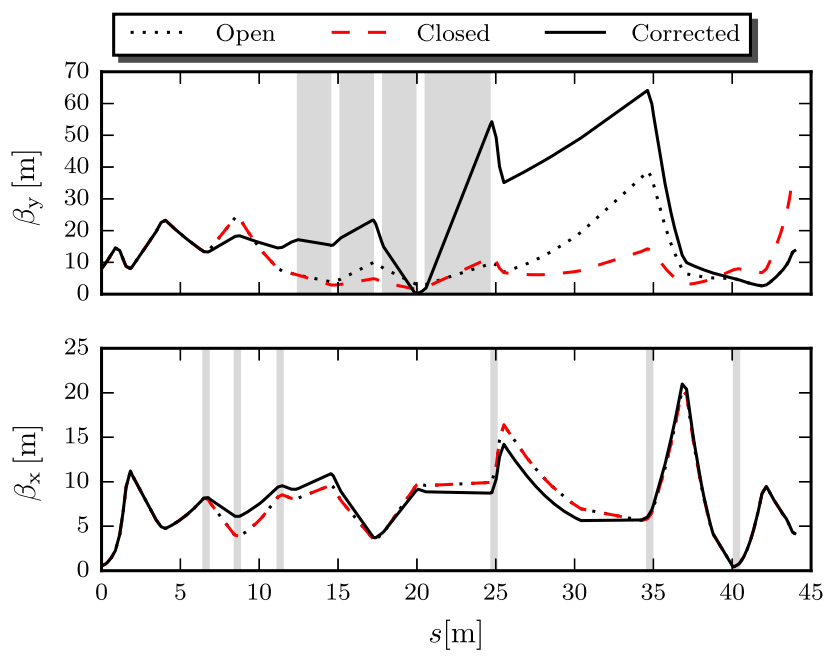

FIG. 6. Evolution of the $\beta$-functions in dependence on the longitudinal coordinate $s$. The undisturbed state (dotted) is compared to the disturbed state with (solid line) and without (dashed) application of the correction. Grey areas in the upper plot mark the position of the variable-gap undulator segments. In this example, a different set of correction magnets is used. Their positions are indicated in the lower plot. Note that at the end of the beam line the corrected optical functions agree with the open case. 
While our method assures the constancy of the transfer matrix between the first and the last corrector magnet, it however makes no statement about the behavior of the optical functions within this correction section. Using the particle tracking code ELEGANT, the optics can be checked for unwanted features and the efficacy of the correction can be verified, see Fig. 6 .

\section{MEASUREMENTS}

Diagnostics in the FLASH1 main undulator area include wire scanners at the ends of the undulator modules [13]. With the help of these devices the beam diameter can be determined along the undulator. The relationship between beam size $\sigma$ and $\beta$-function in the vertical and horizontal direction is given by the well known equation,

$$
\sigma_{u}(s)=\sqrt{\varepsilon_{u} \beta_{u}(s)}, \quad u \in\{x, y\},
$$

where $s$ is the longitudinal position and $\varepsilon_{u}$ denotes the geometric beam emittance. A successful correction can therefore be verified experimentally, by comparing the beam sizes along the undulator.

The measurements presented in Fig. 7 have been conducted with a $1.0-\mathrm{GeV}$-electron beam, while the disturbance was introduced by closing all four seeding undulator segments to their lowest gap value.

As expected, closing the seeding undulator primarily affects the beam size in the vertical direction. After the optics correction is applied, the beam sizes correspond to

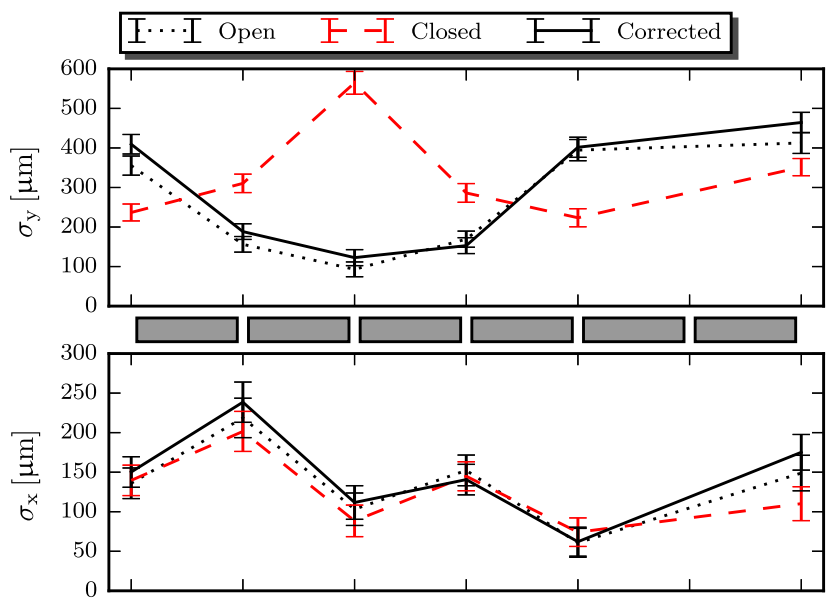

FIG. 7. Transverse beam sizes measured using the wire scanners between the six FLASH main undulator segments (grey boxes in the middle). The dotted curve shows the beam sizes at theory optics without any disturbance. Measurements of the disturbed case, where the seeding undulator is closed, are depicted by the dashed curve. The solid curve shows beam sizes with corrections applied. At the time of measurement, the wire scanner between the last two undulator segments was unavailable. those measured with an open undulator within the range of measuring accuracy. Thereby, it is shown experimentally that our method is able to restore the initial course of the optical functions in the main undulator with satisfactory precision.

As mentioned above, the motivation to develop the presented method was to allow simultaneous operation of both the sFLASH and FLASH1 main undulator. In recent efforts to establish procedures for three beam-line lasing (FLASH1, FLASH2, sFLASH) the described method is in frequent use [14]. Once FLASH1 SASE operation is established, the sFLASH variable-gap undulator segments are closed to their target gap values, causing the SASE signal of the main undulator to vanish. Subsequently, the calculated correction is applied to the chosen corrector quadrupole magnets. This on-line procedure was able to recover $70 \%$ of the initial FEL photon pulse energy with only a few orbit corrections applied. We suspect the drop in FEL power to be mainly caused by the deviation from the initial orbit in the main undulator that is introduced by the steering effects of the seeding undulator as well as the correction quadrupole magnets. The deteriorated overlap between the electron bunch and the FEL pulse can certainly amount for a power loss of this magnitude.

\section{CONCLUSION AND OUTLOOK}

We developed a novel approach to compensate for variations in particle beam optics that would otherwise disturb the optics in other parts of the machine, if left uncorrected. It produces continuous, numerical functions for six machine parameters, compensating the unwanted effect of an arbitrary number of disturbance parameters. Because of the continuity of the correction function, it is suitable to be applied simultaneously to the disturbance. Effectively, this method allows to freely modify the optics locally within a beam-line section, without altering the optics in the rest of the machine.

The method has been implemented at FLASH, with the objective of compensating the influence of the variable-gap undulator used by the sFLASH experiment and is in frequent use. We showed measurements confirming the precise restoration of the initial optics.

A next experimental step will be to apply the correction steadily as the disturbance arises, which in the end enables us to close the seeding undulator and start seeding experiments without any notable changes of electron beam quality in the FLASH1 main undulator. This compensation constitutes an important milestone for the planned simultaneous operation of a seeding experiment at FLASH1. The method will generally be useful for two FELs operated independently of each other in cascaded mode. Ultimately, it will be beneficial for all optics manipulations that need to stay restricted to a local section of a long beam line. 


\section{ACKNOWLEDGMENTS}

We would like to thank all colleagues participating in the FLASH operation. This work has been supported by Federal Ministry of Education and Research of Germany under Contract No. 05K1GU4 and the German Research Foundation program graduate school 1355 . Figures 3, 5, 6 and 7 were created using MATPLOTLIB [15].

[1] S. Redaelli et al., Operation of the betatron squeeze at the LHC, in Proceedings of the 4th International Particle Accelerator Conference, IPAC'13, Shanghai, China (JACoW, Geneva, Switzerland, 2013), TUPFI038.

[2] M. Borland, elegant: A flexible SDDS-compliant code for accelerator Simulation, Advanced Photon Source LS-287 (2000).

[3] H. Grote et al., The MAD-X Program (Methodical Accelerator Design) (CERN, Geneva, 2016).

[4] S. Krantz and H. Parks, The Implicit Function Theorem: History, Theory, and Applications (Birkhäuser, Boston, 2003).

[5] J. Bödewadt et al., Recent results from FEL seeding at FLASH, in Proceedings of the 6th International Particle Accelerator Conference, IPAC'15, Richmond, VA, USA (JACoW, Geneva, Switzerland, 2015), TUBC3.

[6] E. Courant and H. Snyder, Theory of the alternatinggradient synchrotron, Ann. Phys. (N.Y.) 3, 1 (1958).
[7] R. Gilmore, Lie Groups, Lie Algebras, and Some of Their Applications, Dover Books on Mathematics (Dover Publications, New York, 2005).

[8] J. Hale, Ordinary Differential Equations, Pure and Applied Mathematics (Wiley-Interscience, New York, 1969).

[9] M. Vogt et al., Status of the free-electron laser user facility FLASH, in Proceedings of the 5th Inter-national Particle Accelerator Conference, IPAC'14, Dresden, Germany (JACoW, Geneva, Switzerland, 2014), TUOCA02.

[10] M. Quattromini, M. Artioli, E. Di Palma, A. Petralia, and L. Giannessi, Focusing properties of linear undulators, Phys. Rev. ST Accel. Beams 15, 080704 (2012).

[11] M. Tischer et al., Undulators of the sFLASH experiment, in Proceedings of the 1st International Particle Accelerator Conference, IPAC'10, Kyoto, Japan (IPAC'10 OC/ ACFA, Kyoto, Japan, 2010), WEPD014.

[12] Wolfram Research, Inc., Mathematica Version 11.0 (Wolfram Research, Inc., Champaign, Illinois, 2016).

[13] U. Hahn, N. v. Bargen, P. Castro, O. Hensler, S. Karstensen, M. Sachwitz, and H. Thom, Wire scanner system for FLASH at DESY, Nucl. Instrum. Methods Phys. Res., Sect. A 592, 189 (2008).

[14] T. Plath et al., Free-electron laser multiplex driven by a superconducting linear accelerator, J. Synchrotron Radiat. 23, 1070 (2016).

[15] J.D. Hunter, Matplotlib: A 2D graphics environment, Comput. Sci. Eng. 9, 90 (2007). 